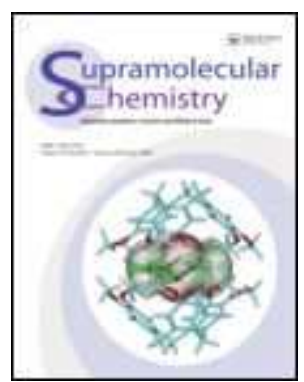

\title{
Fundamental and Applied Aspects of Urea and Thiourea Inclusion Compounds
}

\begin{tabular}{|r|l|}
\hline Journal: & Supramolecular Chemistry \\
\hline Manuscript ID: & GSCH-2006-0030 \\
\hline Manuscript Type: & Review \\
\hline Date Submitted by the & $31-$ May-2006 \\
\hline Complete List of Authors: & Harris, Kenneth; Cardiff University, Chemistry \\
\hline Keywords: & see article \\
\hline
\end{tabular}

\section{S) ScholaroNE \\ Manuscript Central}




\begin{abstract}
$\underline{\text { Abstract }}$
Urea and thiourea inclusion compounds are well known to exhibit a wide range of fundamentally important physico-chemical properties, and through the work of many research groups, these materials have served as model systems for exploring a range of issues of wider relevance to other classes of organic solids. While continuing to investigate these issues, our research in recent years has also focused on issues of a more applied nature, including crystal growth processes, guest exchange processes, the design and development of X-ray dichroic filter materials, and the development of strategies for direct experimental determination of intermolecular interaction energies. This article presents a brief overview of fundamental properties of urea and thiourea inclusion compounds, and reviews some of our recent work in the specific areas identified above.
\end{abstract}

Keywords: urea inclusion compounds; thiourea inclusion compounds; crystal growth; crystal morphology; guest exchange processes; X-ray dichroism; intermolecular interaction energies 


\section{$\underline{\text { Introduction }}$}

There is currently considerable interest in understanding structural, dynamic and chemical properties of solid one-dimensional inclusion compounds, typified by urea and thiourea inclusion compounds [1-5]. In "conventional" urea inclusion compounds, the host structure [6,7] comprises an extensively hydrogen-bonded arrangement of urea molecules, within which there are linear, parallel tunnels (Figure 1). The diameter of the urea tunnel varies between $c a .5 .5 \AA$ and $5.8 \AA$ as a function of position along the tunnel [8], and the dimensions of the tunnel are suitable for accommodating guest molecules based on a sufficiently long n-alkane chain and with a sufficiently low degree of substitution. As discussed in more detail below, the urea tunnel structure is stable only when the tunnels are filled with a dense packing of guest molecules. A wide range of different types of guest molecules have been shown to form urea inclusion compounds, and a notable feature is that the vast majority of these inclusion compounds have the same urea host structure at ambient temperature. We refer to such cases as "conventional" urea inclusion compounds, characterized by: (i) a hexagonal host tunnel structure (space group $\mathrm{P}_{1} 22$ or $\mathrm{P}_{5} 22$ ) [6,7], (ii) an incommensurate relationship [9] between the periodicities of the host and guest substructures along the tunnel axis, and (iii) substantial dynamic disorder (reorientation about the tunnel axis) of the guest molecules at ambient temperature. In most cases, order-disorder phase transitions occur at sufficiently low temperature, and are associated with a distortion of the host tunnel (to a lower symmetry than hexagonal) and a concomitant decrease in the reorientational motion of the guest molecules. A wide range of fundamental physico-chemical properties of urea inclusion compounds have captivated the attentions of many research groups over several years, with particular focus on understanding the incommensurate structural properties [7,10-14], the order-disorder phase transitions [15-20], dynamic properties (particularly concerning molecular motion of the guest molecules) [21-26], properties relating to one-dimensional confinement [27-29], host-guest chiral recognition [30,31] and ferroelastic properties [32,33].

The sulphur analogue of urea - thiourea - also forms solid inclusion compounds based on a tunnel host structure [2,3]. The tunnels in the thiourea host structure are larger in cross-sectional area than those in the urea host structure, and as a consequence, the urea and thiourea host structures tend to incorporate different types of guest molecule. For example, thiourea forms tunnel inclusion compounds with cyclohexane, several derivatives of cyclohexane, ferrocene, other organometallics, 
and certain compounds containing a benzene ring. In general, such guest molecules are too large to be accommodated within the host structure in urea inclusion compounds. For guest molecules with a fairly isotropic shape (e.g. cyclohexane), the host structure at ambient temperature is usually rhombohedral, and the guest molecules are generally disordered. In many cases, this rhombohedral structure undergoes a phase transition to a monoclinic structure at sufficiently low temperature, with concomitant ordering of the guest molecules. For guest molecules that are more planar in shape, on the other hand, the thiourea inclusion compounds tend to have a monoclinic host structure at ambient temperature, and the guest molecules are generally ordered. While the host tunnel in conventional urea inclusion compounds is fairly cylindrical (with only small fluctuations in tunnel diameter on moving along the tunnel), the rhombohedral thiourea tunnel structure has prominent bulges (diameter $\approx 7.1 \AA$ ) and constrictions (diameter $\approx 5.8 \AA$ ) at different positions along the tunnel. As a consequence, many properties of thiourea inclusion compounds are more directly understood by considering the thiourea host structure as "cage" type rather than "tunnel" type.

Much of our own research over several years has focused on understanding a range of physico-chemical properties of urea and thiourea inclusion compounds, recognizing that these materials serve as model systems for exploring a range of fundamental issues of wider relevance to other classes of organic materials. Our early research was focused on understanding periodic structural properties (with particular interest in the experimental assessment and theoretical understanding of incommensurate versus commensurate behaviour), local structural properties (including characterization of intermolecular interactions in disordered systems), dynamic properties, host-guest chiral recognition, and structural and dynamic aspects of order-disorder phase transitions. While continuing to investigate these aspects, our research in recent years has focused increasingly on using the fundamental understanding developed in these earlier studies in order to explore properties of more applied relevance. These recent areas of interest include studies of crystal growth processes, guest exchange processes, X-ray dichroism, and the development of strategies for direct experimental determination of intermolecular interaction energies. This article is focused on highlighting some of these recent areas of interest, with specific reference to our own work in these areas. We recognize, of course, that parallel advances in many aspects of urea and thiourea inclusion compounds have been made by a wide range of other workers in this field, as covered elsewhere in the literature. 


\section{Control of Crystal Morphology}

To explore several fundamental properties of crystalline solids, and to exploit many of their potential applications, it can be crucial to obtain single crystals with a specific desired shape (morphology). However, the crystal morphology produced spontaneously by normal crystal growth procedures is often not the specific morphology that is required for a particular application, and in such cases, experimental strategies must be devised to bias the crystal growth process to produce crystals with the required morphology. Crystal growth is generally governed by kinetic factors, and to alter the crystal morphology requires control of the relative rates of growth of the crystal in different directions (i.e. the relative rates of growth of different crystal faces). For the case of alkane/urea inclusion compounds, spontaneous crystal growth using conventional solution state crystallization procedures generates a long hexagonal needle morphology (with the tunnel axis parallel to the needle axis), as the rate of growth parallel to the tunnel (denoted $k_{\|}$) is substantially greater than the rate of growth perpendicular to the tunnel (denoted $k_{\perp}$ ). For certain applications of urea inclusion compounds, however, we may require that the crystals have a hexagonal flat-plate morphology (with the tunnel axis perpendicular to the plane of the plate). Our work in this area has focused on the development of a general strategy for controlling the crystal morphology of tunnel inclusion compounds (within the full range from long needle crystals to flat plate crystals) [34,35], and has demonstrated the successful application of this strategy in the case of alkane/urea inclusion compounds. Initially, it was shown [34] that, by using a selective crystal growth inhibitor (5octadecyloxyisophthalic acid) that reduces the rate of crystal growth along the tunnel direction (such that $k_{\|}<<k_{\perp}$ ), crystals of alkane/urea inclusion compounds can be induced to grow as hexagonal flat plates, rather than the long-needle crystals obtained under normal crystal growth conditions (in the absence of the crystal growth inhibitor). Subsequently, we demonstrated [35] that, by altering the concentration $\left(c_{\mathrm{inh}}\right)$ of the crystal growth inhibitor in the crystallization solution, crystals with any desired morphology could be grown (see Figure 2) between the two extreme situations of long needle crystals $\left(c_{\text {inh }}=0 ; k_{\|}>>k_{\perp}\right)$ and flat plate crystals $\left(c_{\text {inh }}\right.$ sufficiently large; $\left.k_{\|}<<k_{\perp}\right)$, including crystals that have comparable dimensions parallel and perpendicular to the tunnel axis (intermediate values of $\left.c_{\text {inh }} ; k_{\|} \approx k_{\perp}\right)$. 


\section{Guest Exchange Processes}

Many different types of solid inclusion compounds (e.g. zeolites) have found important applications in molecular separation processes, based on the fact that the host structure displays selectivity with regard to the incorporation of guest molecules of differing size and shape. In the case of urea inclusion compounds, however, the fact that the "empty" urea tunnel structure is unstable [36] limits the opportunity to develop potential applications based on molecular adsorption and/or molecular separation within the empty host structure, analogous to the types of process that have been exploited for zeolitic host materials. Nevertheless, a process for achieving guest exchange in urea inclusion compounds, by a mechanism that does not proceed via the empty host tunnel structure, has been identified [28]. This mechanism for guest exchange is based on the requirement that the tunnels remain fully occupied by guest molecules at all stages throughout the guest exchange process, but with the actual identity of the guest molecules changing as a function of time during the process. Thus, it has been demonstrated [28] that net transport of guest molecules in one direction along the tunnel in the urea host structure can be achieved by inserting "new" (thermodynamically more favourable) guest molecules at one end of a crystal of a urea inclusion compound (e.g. by dipping the crystal into the liquid phase of the new guest), with the "original" guest molecules expelled from the other end of the crystal.

To obtain deeper insights into such guest exchange processes in urea inclusion compounds, we have used confocal Raman microspectrometry as an in situ probe (Figure 3a), demonstrating [37] that this technique can yield information on the spatial distribution of the original and new guest molecules within the crystal, and details of how the spatial distribution of the original and new guest molecules varies as a function of time during the process. Our work in this area has focused primarily on the system comprising the 1,8-dibromooctane/urea inclusion compound as the original crystal, and pentadecane as the new type of guest molecule. Analysis of the Raman microspectrometry data has focused [37] on studying the variation of the intensity of the $\mathrm{C}-\mathrm{Br}$ stretching band of the original 1,8-dibromooctane guest molecules (for the predominant trans endgroup conformation), as a function of position in the crystal and as a function of time (Figure $3 \mathrm{~b}$ ). Clearly, such data provide access to quantitative information on kinetic and mechanistic aspects of the transport of guest molecules through the host tunnel structure during the guest exchange process. For example, our in situ studies employing confocal Raman microspectrometry [38] have 
revealed that the guest exchange process is associated with significant changes in the conformational properties of the original (1,8-dibromooctane) guest molecules, corresponding to a significant increase in the proportion of 1,8-dibromooctane guest molecules with the gauche endgroup conformation within the "boundary region" between the original and new guest molecules. At this stage, several fundamental aspects relating to such guest exchange processes remain to be understood, and a variety of techniques are currently being employed for this purpose. Clearly, the development of an understanding of the fundamentals of the guest exchange process in such materials is a pre-requisite for developing and optimizing a range of potential applications in molecular separation, based for example on discrimination of molecular size, shape and chirality.

\section{Crystals as Dichroic Filters for X-ray Polarization Analysis}

In the case of visible light, studies of polarization properties are dominated by the use of dichroic filters (e.g. the Polaroid sheet), for which radiation with polarization parallel to a certain axis in the dichroic filter material is preferentially attenuated over radiation with polarization perpendicular to this axis. In principle, materials analogous to the Polaroid sheet may be developed as dichroic filters for polarized X-ray radiation, and such materials have considerable potential in a range of applications (e.g. in the field of magnetic X-ray scattering). However, the development of a dichroic filter material for X-ray polarization analysis was demonstrated for the first time only recently [39]. The material studied was the urea inclusion compound containing 1,10-dibromodecane $\left[\mathrm{Br}\left(\mathrm{CH}_{2}\right)_{10} \mathrm{Br}\right]$ guest molecules, and the successful application of this material as a dichroic filter was demonstrated (at X-ray energies near the bromine K-edge) in a study of magnetic diffraction from antiferromagnetic holmium. In spite of this success, however, the efficiency of the 1,10dibromodecane/urea inclusion compound as a dichroic filter is limited by the fact that the $\mathrm{C}-\mathrm{Br}$ bonds in the guest molecule undergo reorientation around the tunnel axis at ambient temperature and are oriented at $c a .35^{\circ}$ to this axis. Theoretical considerations, elaborated elsewhere [39], suggested that a material in which all $\mathrm{C}-\mathrm{Br}$ bonds are aligned parallel to the tunnel would be significantly more effective than 1,10-dibromodecane/urea as an X-ray dichroic filter, and this theoretical prediction provided the motivation for the design and preparation of a new material exhibiting the required structural properties. The "crystal design" strategy converged on the proposal [40] that an inclusion compound comprising 1-bromoadamantane guest molecules within the 
thiourea host structure would have the $\mathrm{C}-\mathrm{Br}$ bonds of all 1-bromoadamantane guest molecules aligned parallel to the tunnel direction (constrained by the orienting influence of the thiourea host structure), and hence parallel to each other, as required for an X-ray dichroic filter material of improved efficiency. Following the preparation of this material, structure determination from single crystal X-ray diffraction data (Figure 4a) showed [40] that the design strategy was indeed vindicated, with the $\mathrm{C}-\mathrm{Br}$ bonds of all 1-bromoadamantane guest molecules aligned parallel to the tunnel axis (Figure 4b). In this inclusion compound, the host structure differs to some extent from the conventional thiourea host structure. In particular, within the repeat distance $(24.75 \AA)$ along the tunnel, three crystallographically independent sites are occupied by the 1-bromoadamantane guest molecules, corresponding to a guest/thiourea molar ratio of $1 / 4$. At each site, there is disorder between two orientations (with essentially equal occupancies) of the guest molecule, but importantly, the $\mathrm{C}-\mathrm{Br}$ bond of the guest molecule in each orientation lies parallel to the thiourea host tunnel.

To assess the performance of the 1-bromoadamantane/thiourea inclusion compound as a dichroic filter material, X-ray dichroism spectra were measured [40] for a single crystal on Station 16.3 at the Synchrotron Radiation Source, Daresbury Laboratory. Figure 4c shows the X-ray transmittance as a function of X-ray energy (close to the bromine K-edge at $13.474 \mathrm{keV}$ ) for both the parallel and perpendicular orientations of the crystal, and Figure $4 \mathrm{~d}$ shows the X-ray transmittance as a function of crystal orientation at $13.479 \mathrm{keV}$. Close to the absorption edge, the transmission of polarized X-rays through the crystal shows a very significant dependence on crystal orientation. The maximum dependence is at $13.479 \mathrm{keV}$, and corresponds to a value of $R_{\gamma}=0.32$ (where $R_{\gamma}$ is defined as $\gamma_{\min } / \gamma_{\max }$, and $\gamma_{\min }$ and $\gamma_{\max }$ are the values of the linear absorption coefficient for the crystal orientations with lowest and highest attenuation respectively). This value of $R_{\gamma}$ is very close to the optimal (i.e. minimum) attainable value predicted theoretically [39].

As discussed above, X-ray dichroic filter materials have important (but as yet unexploited) potential applications in X-ray polarimetry, including their use in polarization analysis of magnetic $\mathrm{X}$-ray scattering and in X-ray astronomy, as elaborated in more detail elsewhere [41]. 


\section{$\underline{\text { Experimental Assessment of Host-Guest Interaction Energies }}$}

In order to derive a fundamental rationalization of the structural properties of molecular solids, it is essential to understand the intermolecular interactions that govern the observed molecular packing arrangement. With this motivation, a strategy has been developed [42] for using incommensurate [9] solid inclusion compounds based on one-dimensional tunnel structures as a system for estimating intermolecular interaction energies (particularly relating to host-guest interaction) directly from experimental investigations. The method focuses on the competitive co-inclusion of two different types of guest molecule $\mathrm{X}(\mathrm{S})_{\mathrm{n}_{\mathrm{i}}} \mathrm{X}$ and $\mathrm{X}(\mathrm{S})_{\mathrm{n}_{\mathrm{j}}} \mathrm{X}$ within the same host tunnel structure, where $\mathrm{X}$ represents an appropriate end-group, $\mathrm{S}$ represents an appropriate spacer unit, and $n_{\mathrm{i}} \neq n_{\mathrm{j}}$. For each pair of guest molecule types $(i, j)$, the inclusion compound is prepared for several different values of the proportion of $\mathrm{X}(\mathrm{S})_{\mathrm{n}_{\mathrm{i}}} \mathrm{X}$ molecules in the crystallization solution (denoted $\gamma_{\mathrm{i}}$ ), and in each case the proportion of $\mathrm{X}(\mathrm{S})_{\mathrm{n}_{\mathrm{i}}} \mathrm{X}$ guest molecules in the resultant inclusion compound (denoted $\left.m_{\mathrm{i}}\right)$ is measured. Thus, the experimental data comprise several values of $\left(\gamma_{\mathrm{i}}, m_{\mathrm{i}}\right)$ for each pair of guest molecule types $(i, j)$. The values of $\left(\gamma_{\mathrm{i}}, m_{\mathrm{i}}\right)$ indicate the relative extent of uptake of the two different types of guest molecule within the host tunnel structure, which in turn depends on the intermolecular interaction energies within the resultant inclusion compound. In particular, given that the two different types of guest molecule $X(S)_{n_{i}} X$ and $X(S)_{n_{j}} X$ have the same end-groups $X$, the relative extent of uptake of the two types of guest molecule within the inclusion compound is dominated by the influence of host-guest interaction (which differs for the two types of guest molecule as they contain a different number of spacer units $S$ ), rather than guest-guest interaction (which is the same $\mathrm{X} \cdots \mathrm{X}$ interaction between each pair of guest molecule types). A theoretical model has been developed [42] for determining host-guest interaction energy terms directly from experimentally measured $\left(\gamma_{\mathrm{i}}, m_{\mathrm{i}}\right)$ data, and this methodology has been applied [43] to real experimental data for urea inclusion compounds containing binary mixtures of $\alpha, \omega$-dibromoalkane $\left[\mathrm{Br}\left(\mathrm{CH}_{2}\right)_{\mathrm{n}} \mathrm{Br}\right]$ guest molecules. It is found that the interaction energy terms derived from the experimental data are in excellent agreement with the corresponding energy terms computed using a standard potential energy parameterization. 


\section{Concluding Remarks}

As discussed in this article, the fact that urea and thiourea inclusion compounds display a wide range of interesting fundamental physico-chemical properties is well established. Given the vast amount of fundamental information that has now been accumulated for these two families of solid inclusion compounds, there is considerable scope to develop and advance potential applications of these materials in a range of scientific fields, and significant future developments in this direction may be anticipated with confidence.

\section{$\underline{\text { Acknowledgements }}$}

I am grateful to the members of my research group and research collaborators who have made substantial contributions to our research in the specific areas covered by this article (particularly Dr M.-H. Chao, Dr S.P. Collins, Dr A. Desmedt, Dr F. Guillaume, Dr P.E. Jupp, Dr B.M. Kariuki, Ms N.E. Kelly, Dr S.-O. Lee, Dr J. Martí-Rujas, and others mentioned in the references cited). I am also grateful to the funding agencies (particularly E.P.S.R.C.) that have supported our research in this field.

\section{$\underline{\text { References }}$}

1 Fetterly, L.C. Non-Stoichiometric Compounds (Ed: L. Mandelcorn), Academic Press, New York, 1964, p. 491.

2 Takemoto, K.; Sonoda, N. Inclusion Compounds (Eds: J.L. Atwood, J.E.D. Davies, D.D.; MacNicol), Academic Press, New York, 1984, Volume 2, p. 47.

3 Hollingsworth, M.D.; Harris, K.D.M. Comprehensive Supramolecular Chemistry (Eds: D.D. MacNicol, F. Toda, R. Bishop), Pergamon Press, 1996, Volume 6, pp. 177-238.

4 Harris, K.D.M. Chem. Soc. Rev. 1997, 26, 279.

5 Guillaume, F. J. Chim. Phys. (Paris) 1999, 96, 1295.

6 Smith, A.E. Acta Crystallogr. 1952, 5, 224.

7 Harris, K.D.M.; Thomas, J.M. J. Chem. Soc. Faraday Trans. 1990, 86, 2985.

8 George, A.R.; Harris, K.D.M. J. Mol. Graphics 1995, 13, 138.

9 The structural relationship between the periodicity (denoted $c_{\mathrm{h}}$ ) of the host structure along the tunnel and the periodicity (denoted $c_{\mathrm{g}}$ ) of the guest molecules along the tunnel is incommensurate if there are no sufficiently small integers $m$ and $n$ that satisfy the relationship: $m c_{\mathrm{g}}=n c_{\mathrm{h}}$. Thus, the ratio $c_{\mathrm{g}} / c_{\mathrm{h}}$ is not equal to a rational number with sufficiently small denominator. The nature of incommensurate versus commensurate behaviour in tunnel inclusion compounds is discussed in more detail in refs. 10 and 11. 
10 Rennie, A.J.O.; Harris, K.D.M. Proc. Royal Soc. A 1990, 430, 615.

11 Rennie, A.J.O.; Harris, K.D.M. J. Chem. Phys. 1992, 96, 7117.

12 Harris, K.D.M.; Hollingsworth, M.D. Proc. Royal Soc. A 1990, 431, 245.

13 Lefort, R.; Etrillard, J.; Toudic, B.; Guillaume, F.; Breczewski, T.; Bourges, P. Phys. Rev. Lett. 1996, 77, 4027.

14 Weber, T.; Boysen, H.; Frey, F.; Neder, R.B. Acta Crystallogr. 1997, B53, 544.

15 Parsonage, N.G.; Pemberton, R.C. Trans. Faraday Soc. 1967, 63, 311.

16 Chatani, Y.; Taki, Y.; Tadokoro, H. Acta Crystallogr. 1977, B33, 309.

17 Fukao, K. J. Chem. Phys. 1990, 92, 6867.

18 Lynden-Bell, R.M. Mol. Phys. 1993, 79, 313.

19 Yeo, L.; Kariuki, B.M.; Serrano-González, H.; Harris, K.D.M. J. Phys. Chem. B 1997, 101, 9926.

20 Le Lann, H.; Odin, C.; Toudic, B.; Ameline, J.C.; Gallier, J.; Guillaume, F.; Breczewski, T. Phys. Rev. B 2000, 62, 5442.

21 Casal, H.L.; Cameron, D.G.; Kelusky, E.C. J. Chem. Phys. 1984, 80, 1407.

22 Harris, K.D.M.; Jonsen, P. Chem. Phys. Lett. 1989, 154, 593.

23 El Baghdadi, A.; Dufourc, E.J.; Guillaume, F. J. Phys. Chem. 1996, 100, 1746.

24 Guillaume, F.; Sourisseau, C.; Dianoux, A.-J. J. Chim. Phys. (Paris) 1991, 88, 1721.

25 Smart, S.P.; Guillaume, F.; Harris, K.D.M.; Dianoux, A.-J. J. Phys.: Condens. Matter 1994, 6, 2169.

26 Girard, P.; Aliev, A.E.; Guillaume, F.; Harris, K.D.M.; Hollingsworth, M.D.; Dianoux, A.-J.; Jonsen, P. J. Chem. Phys. 1998, 109, 4078.

27 Hollingsworth, M.D.; Cyr, N. Mol. Cryst. Liq. Cryst. 1990, 187, 135.

28 Khan, A.A.; Bramwell, S.T.; Harris, K.D.M.; Kariuki, B.M.; Truter, M.R. Chem. Phys. Lett. 1999, 307, 320.

29 Chao, M.-H.; Harris, K.D.M.; Kariuki, B.M.; Bauer, C.L.; Foxman, B.M. J. Phys. Chem. B 2002, 106, 4032.

30 Arad-Yellin, R.; Green, B.S.; Knossow, M.; Tsoucaris, G. in Inclusion Compounds (Editors: J.L. Atwood, J.E.D. Davies, D.D. MacNicol), Volume 3, Academic Press, New York, 1984, p. 263.

31 Yeo, L.; Harris, K.D.M. J. Chem. Soc., Faraday Trans. 1998, 94, 1633.

32 Brown, M.E.; Hollingsworth, M.D. Nature 1995, 376, 323.

33 Hollingsworth, M.D.; Peterson, M.L.; Rush, J.R.; Brown, M.E.; Abel, M.J.; Black, A.A.; Dudley, M.; Raghothamachar, B.; Werner-Zwanziger, U.; Still, E.J.; Vanecko, J.A. Cryst. Growth Des. 2005, 5, 2100.

34 Lee, S.-O.; Harris, K.D.M. Chem. Phys. Lett. 1999, 307, 327.

35 Kelly, N.E.; Lee, S.-O.; Harris, K.D.M. J. Am. Chem. Soc. 2001, 123, 12682.

36 Removal of guest molecules from urea inclusion compounds leads to the instantaneous collapse of the "empty" tunnel structure to form a structure of higher density (the well-known crystal structure of "pure" urea), which does not contain empty tunnels. 
37 Marti-Rujas, J.; Desmedt, A.; Harris, K.D.M.; Guillaume, F. J. Am. Chem. Soc. 2004, 126, 11124.

38 Martí-Rujas, J.; Harris, K.D.M.; Desmedt, A.; Guillaume, F. J. Phys. Chem. B 2006, 110, 10708.

39 Collins, S.P.; Laundy, D.; Harris, K.D.M.; Kariuki, B.M.; Bauer, C.L.; Brown, S.D.; Thompson, P. J. Phys.: Condens. Matter 2002, 14, 123.

40 Chao, M.-H.; Kariuki, B.M.; Harris, K.D.M.; Collins, S.P.; Laundy, D. Angew. Chemie Int. Ed. 2003, 42, 2982.

41 Bannister, N.P.; Harris, K.D.M.; Collins, S.P.; Martindale, A.; Solan, G.; Monks, P.; Fraser, G.W. Experimental Astronomy, in press.

42 Harris, K.D.M.; Jupp, P.E.; Lee, S.-O. J. Chem. Phys. 1999, 111, 9784.

43 Lee, S.-O.; Harris, K.D.M.; Jupp, P.E.; Yeo, L. J. Am. Chem. Soc. 2001, 123, 12913.

\section{Figure Captions}

Figure 1 Structure of the hexadecane/urea inclusion compound at ambient temperature, showing nine complete tunnels (with van der Waals radii) viewed along the tunnel axis. The guest molecules have been inserted into the tunnels illustrating orientational disorder, in conformity with the observed X-ray diffraction data and results from spectroscopic investigations.

Figure 2 Optical micrographs (scale divisions $=1 \mathrm{~mm}$ ) of crystals of the hexadecane/urea inclusion compound grown: (a) under conventional conditions with $c_{\text {inh }}=0 \%$ (from top: $\left.R=0.029, R_{\mathrm{m}}=-0.94 ; R=0.035, R_{\mathrm{m}}=-0.93 ; R=0.045, R_{\mathrm{m}}=-0.91\right)$, (b) with $c_{\text {inh }}$ $=3 \%\left(R=1.41, R_{\mathrm{m}}=+0.17\right)$, (c) with $c_{\mathrm{inh}}=5 \%\left(R=8.60, R_{\mathrm{m}}=+0.79\right)$. In each of (b) and (c), the same crystal is viewed parallel (left side) and perpendicular (right side) to the tunnel direction. Aspect ratios are defined as $R=W / L$ (where $L$ is the length of the crystal along the tunnel direction and $W$ is the width of the crystal perpendicular to the tunnel direction), and $R_{\mathrm{m}}=(R-1) /(R+1)$. Note that $R_{\mathrm{m}} \rightarrow-1$ for long-needle crystals, and $R_{\mathrm{m}} \rightarrow+1$ for flat-plate crystals.

Figure 3 (a) Schematic representation of the experimental assembly for in situ Raman microspectrometry of guest exchange in a urea inclusion compound, comprising the single crystal of the urea inclusion compound (green), initially containing 1,8dibromooctane guest molecules, attached to a reservoir containing liquid pentadecane.

(b) In situ time-resolved and spatially resolved monitoring of guest exchange in a single crystal of a urea inclusion compound, using Raman microspectrometry [37]. The Raman micrographs were recorded during transport of pentadecane molecules into and along the tunnels, displacing the guest molecules (1,8-dibromooctane) originally present. The probed region shown represents only part of the crystal, and the transport of guest molecules occurs from left to right (the tunnels run horizontally in the micrographs shown). Regions coloured blue are rich in pentadecane, and regions coloured green are 
rich in 1,8-dibromooctane. The time taken to record each micrograph was $\mathrm{ca} .28$ minutes; the three micrographs shown were recorded (a) 18 hours, (b) 29 hours, and (c) 40 hours after commencement of the guest exchange process.

Figure 4 (a) Crystal structure of the 1-bromoadamantane/thiourea inclusion compound viewed along the tunnel axis of the thiourea host structure.

(b) Crystal structure of 1-bromoadamantane/thiourea viewed perpendicular to the tunnel axis (vertical), showing that the $\mathrm{C}-\mathrm{Br}$ bonds of the 1-bromoadamantane guest molecules lie parallel to the tunnel axis (only one component of the disordered guest substructure is shown).

(c) X-ray transmittance $\left(I / I_{\mathrm{o}}\right)$ through a single crystal of 1-bromoadamantane/thiourea as a function of energy close to the bromine K-edge, with the X-ray polarization parallel $\left(0^{\circ}\right.$ and $\left.180^{\circ}\right)$ and perpendicular $\left(90^{\circ}\right.$ and $\left.270^{\circ}\right)$ to the tunnel axis. The vertical line is at $13.479 \mathrm{keV}$ (see text).

(d) X-ray transmittance $\left(I / I_{\mathrm{o}}\right)$ as a function of the orientation of a single crystal of 1bromoadamantane/thiourea, with the orientation defined as the angle between the tunnel axis ( $\mathrm{C}-\mathrm{Br}$ bond direction) and the electric vector of the polarized incident $\mathrm{X}$-ray beam, at the value of energy $(13.479 \mathrm{keV})$ at which the ratio of absorption coefficients $R_{\gamma}$ is minimum. 


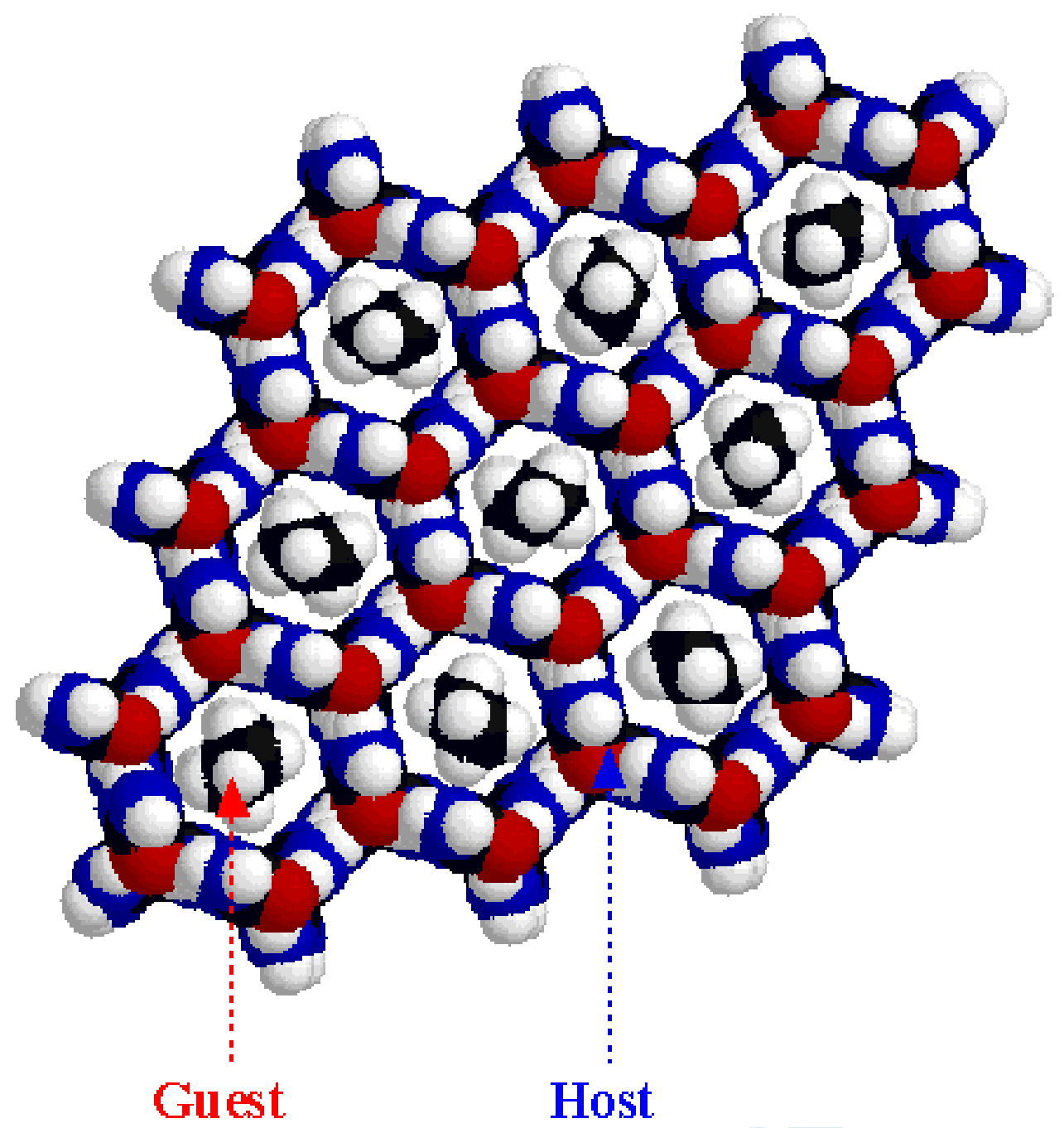

Figure 1 
(a)

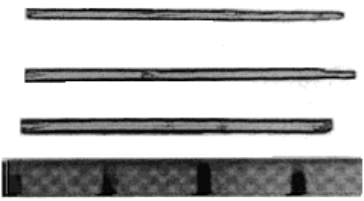

(b)

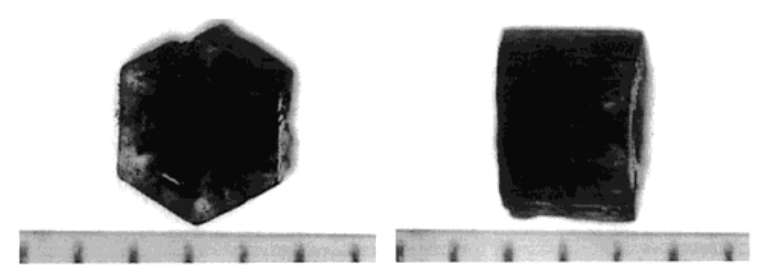

(c)

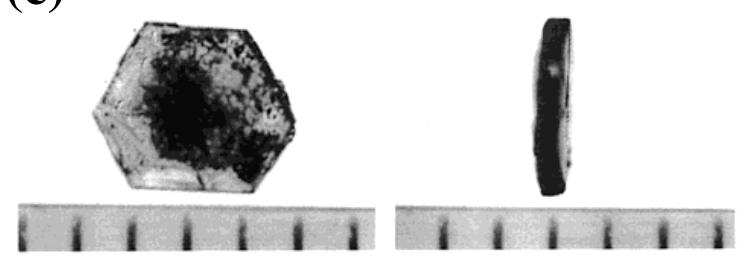

Figure 2 


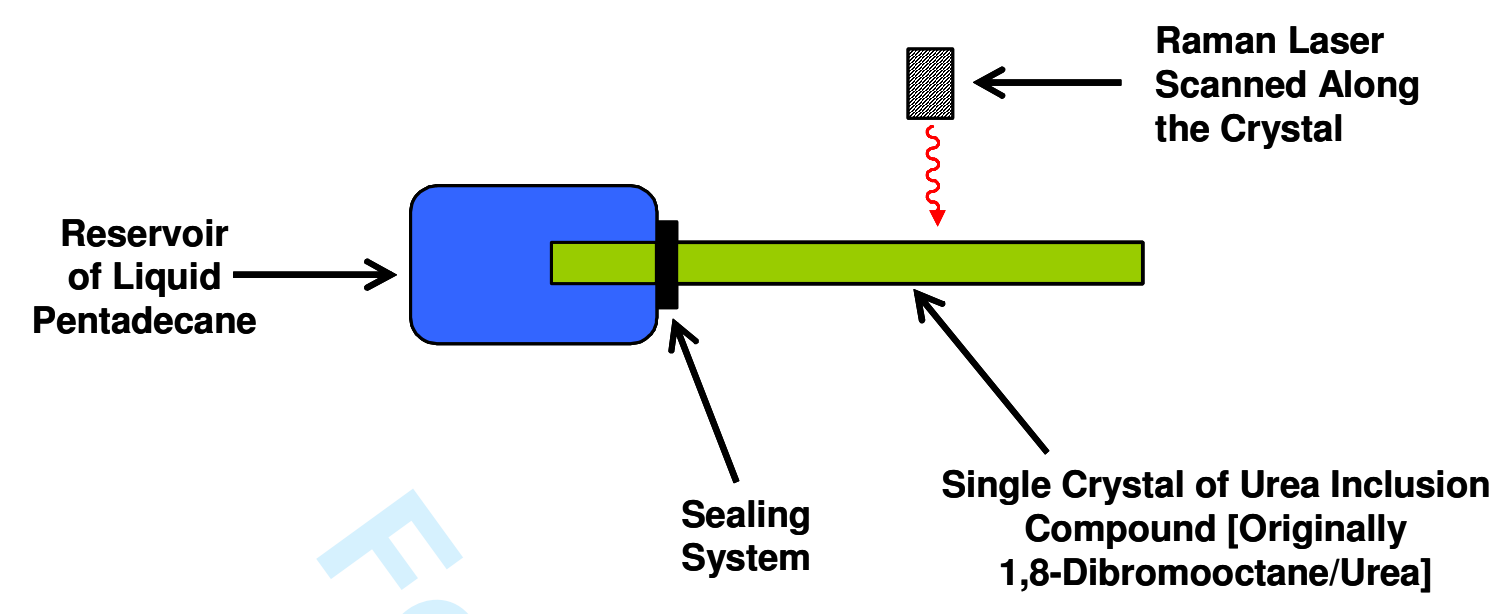

Figure 3(a)

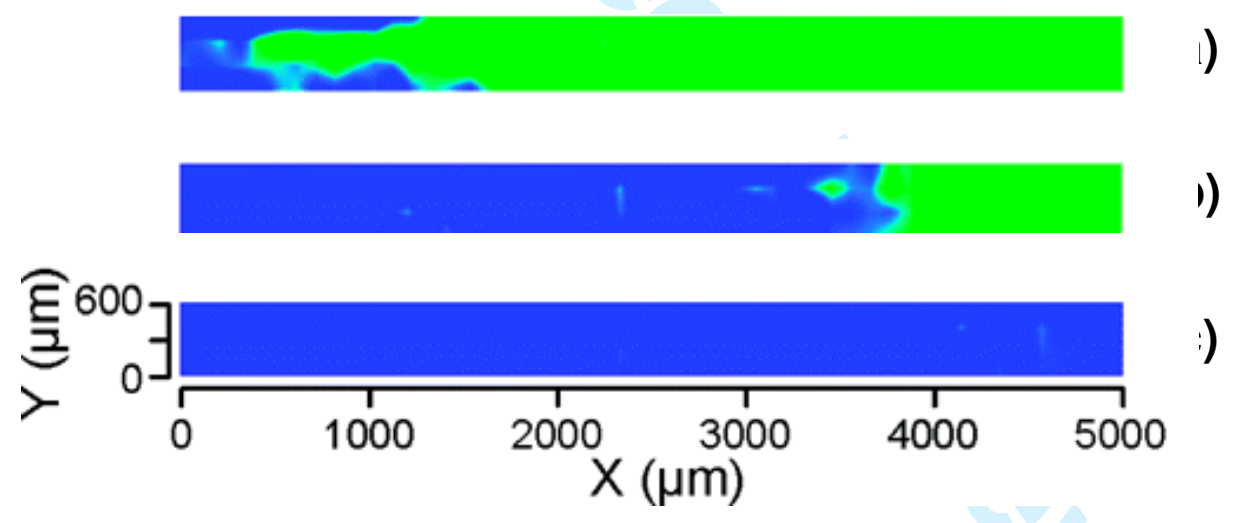

Figure 3(b) 


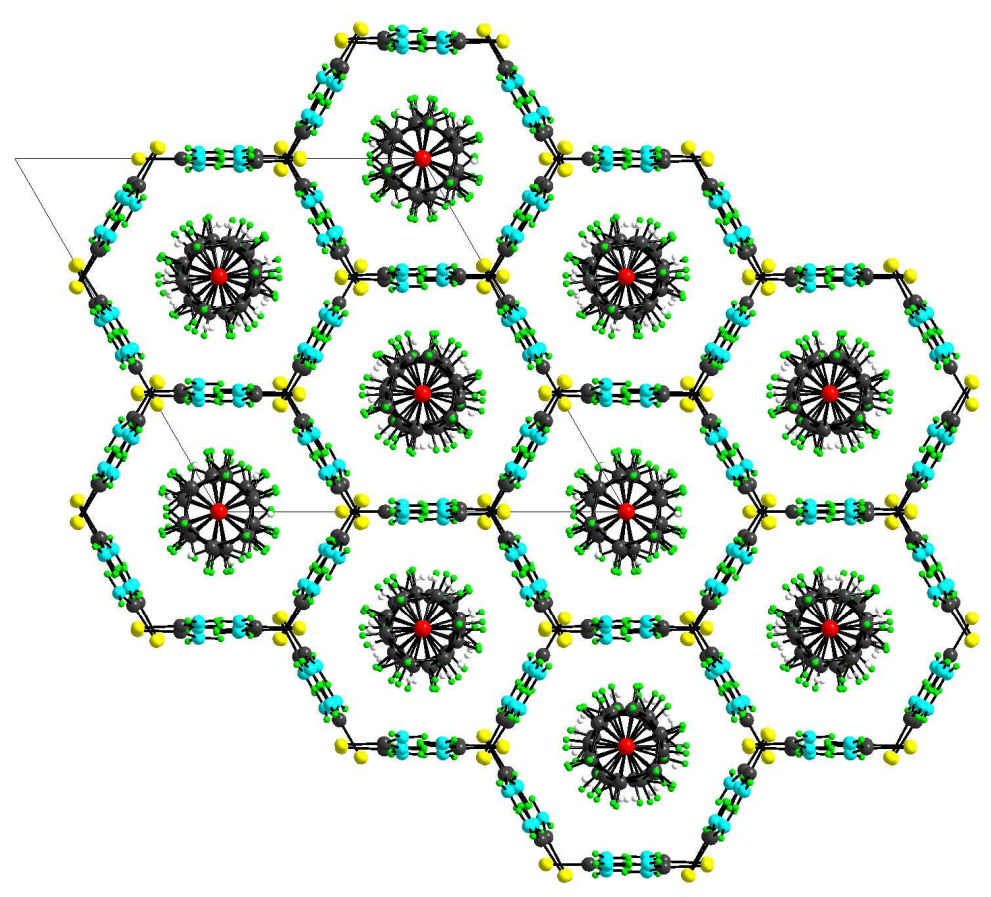

\section{Figure 4(a)}

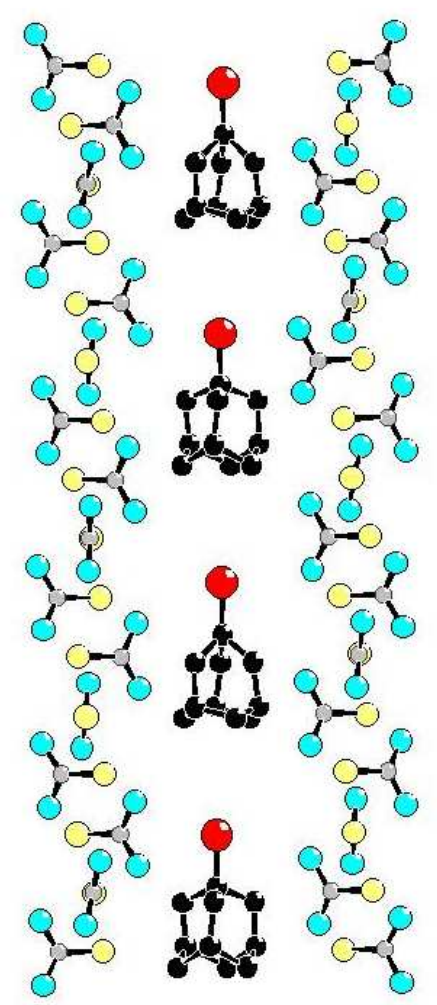

$\underline{\text { Figure 4(b) }}$ 


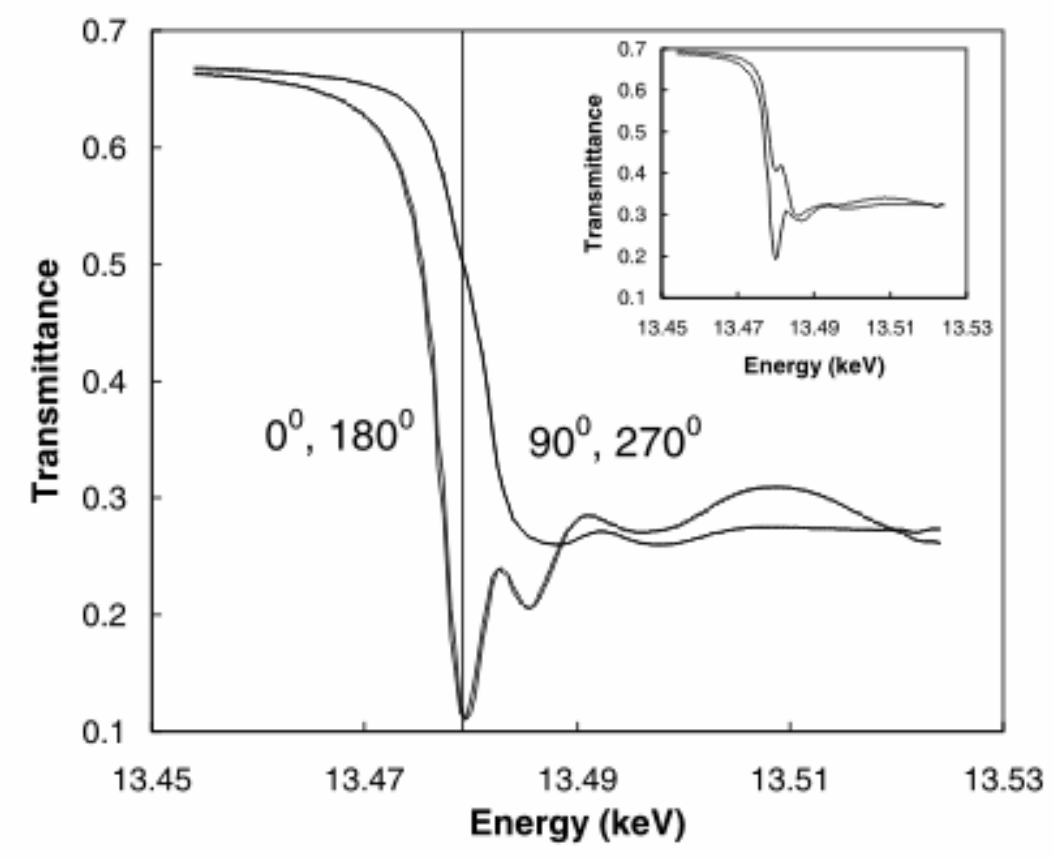

Figure 4(c)

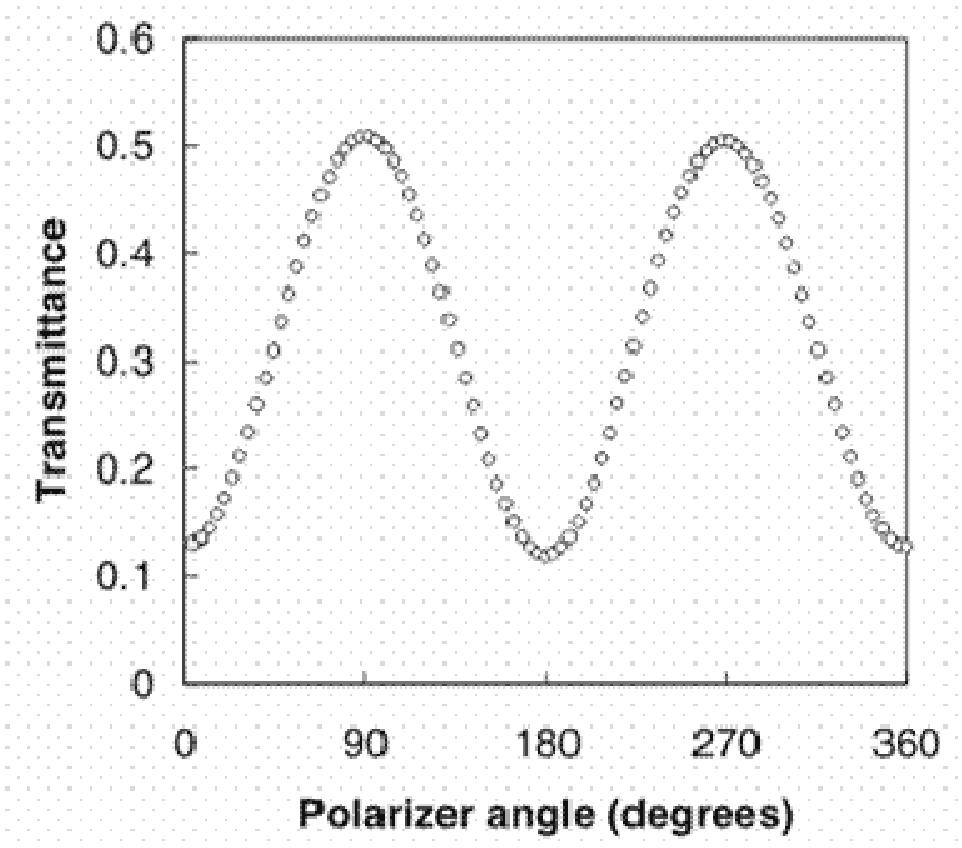

$\underline{\text { Figure 4(d) }}$ 


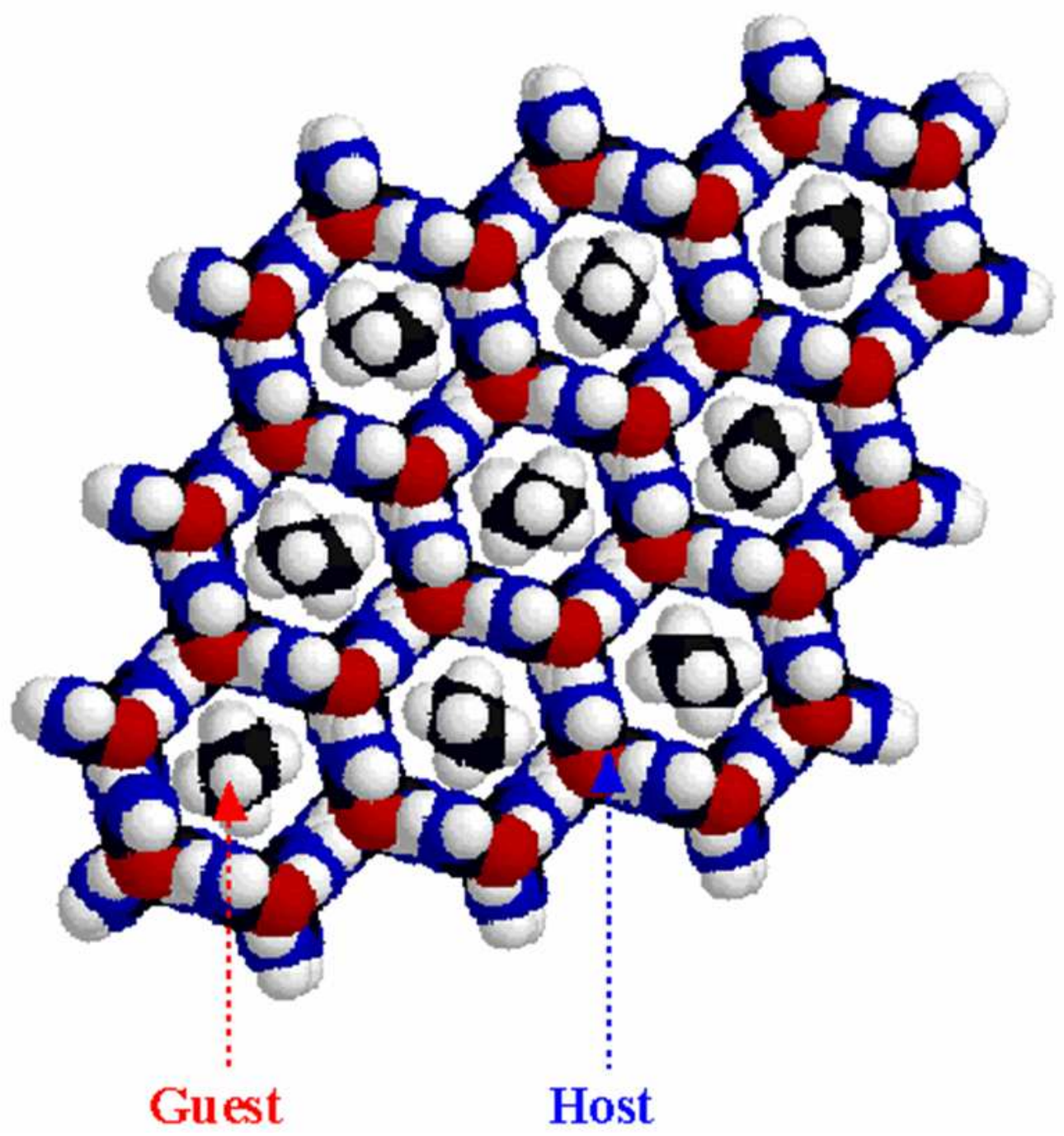

$131 \times 139 m m(150 \times 150$ DPI $)$ 

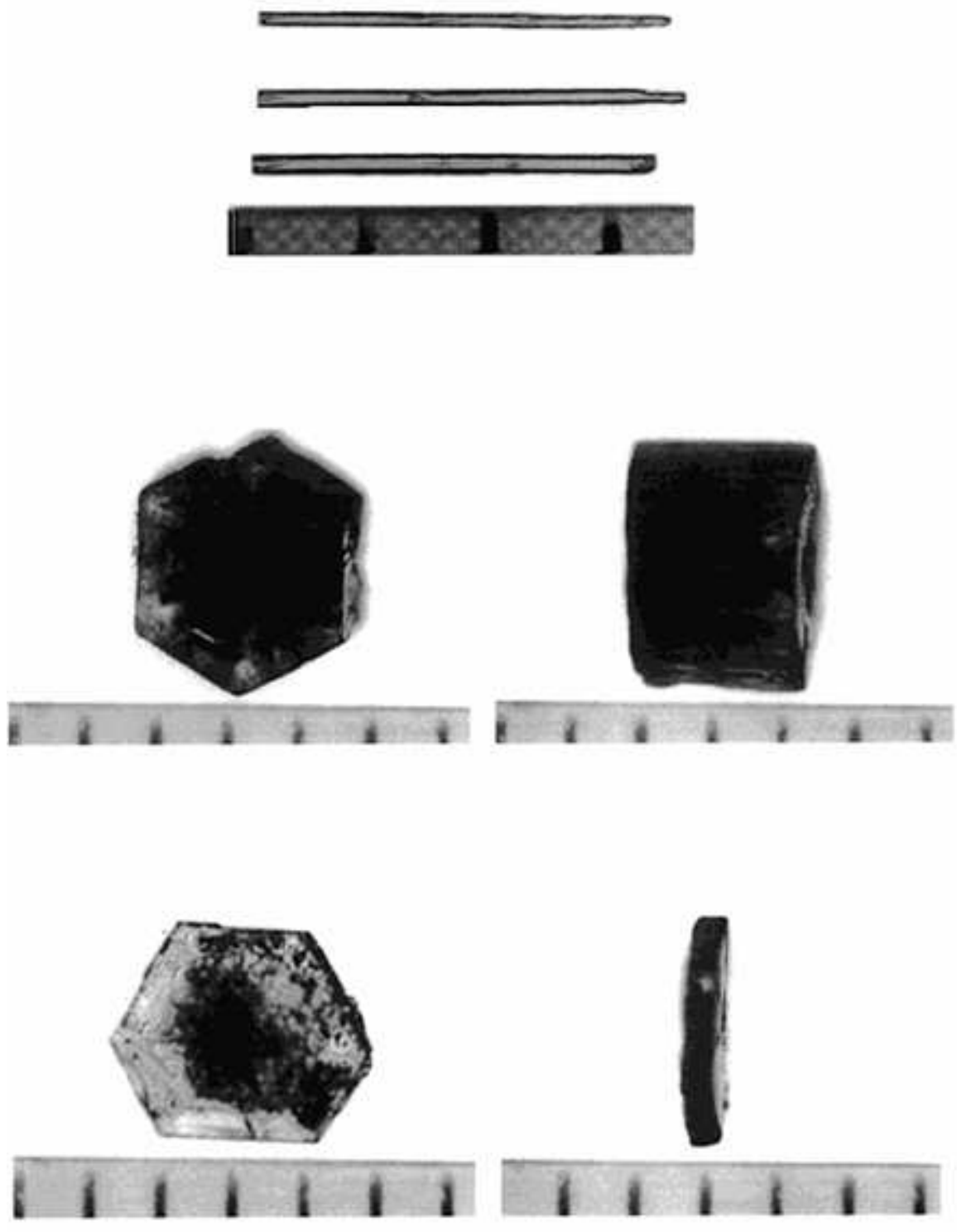

76×99mm (150 x 150 DPI $)$

URL: http:/mc.manuscriptcentral.com/tandf/gsch Email: suprachem@mail.cm.utexas.edu 


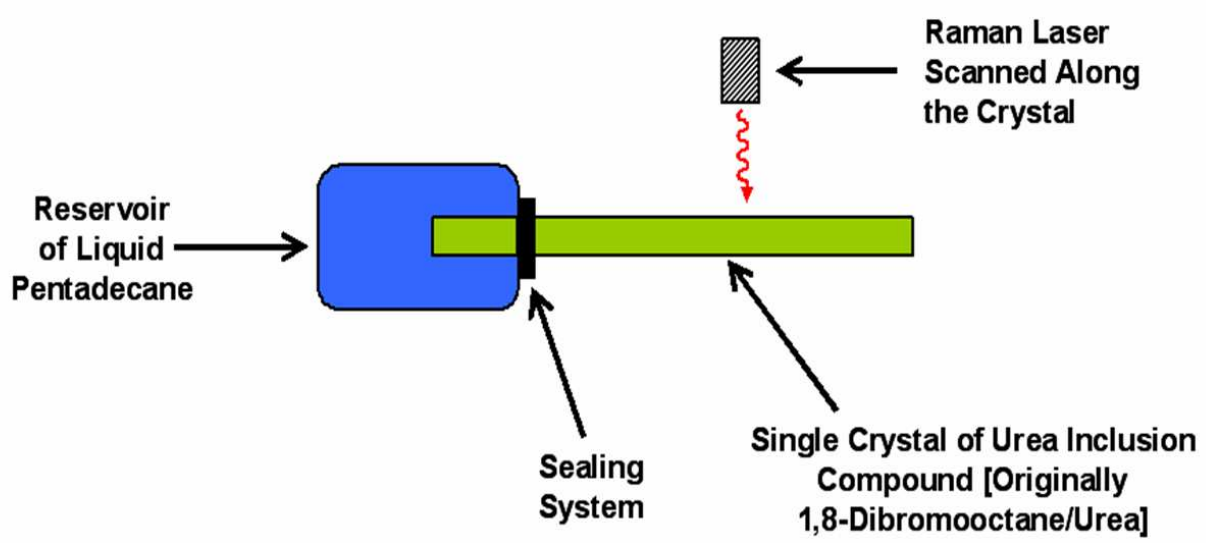

$244 \times 106 \mathrm{~mm}(133 \times 150 \mathrm{DPI})$ 


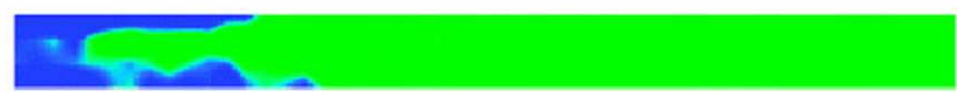

(a)

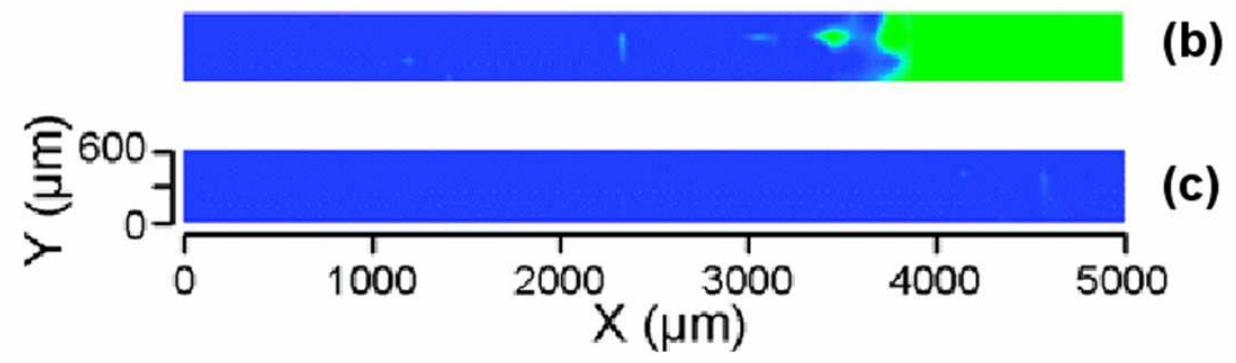

$196 \times 88 \mathrm{~mm}(150 \times 150 \mathrm{DPI})$ 


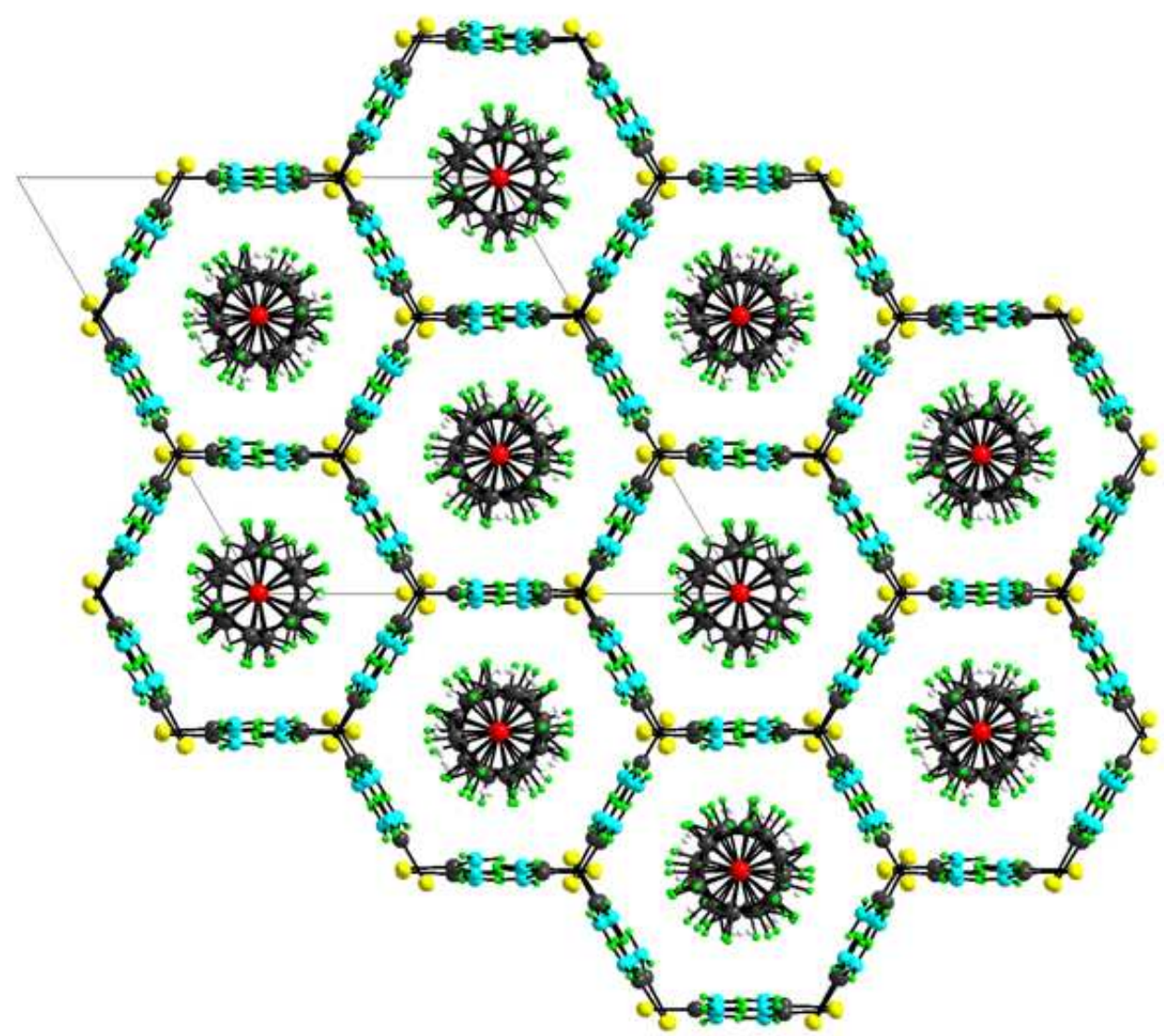

$103 \times 94 \mathrm{~mm}(150 \times 150 \mathrm{DPI})$ 


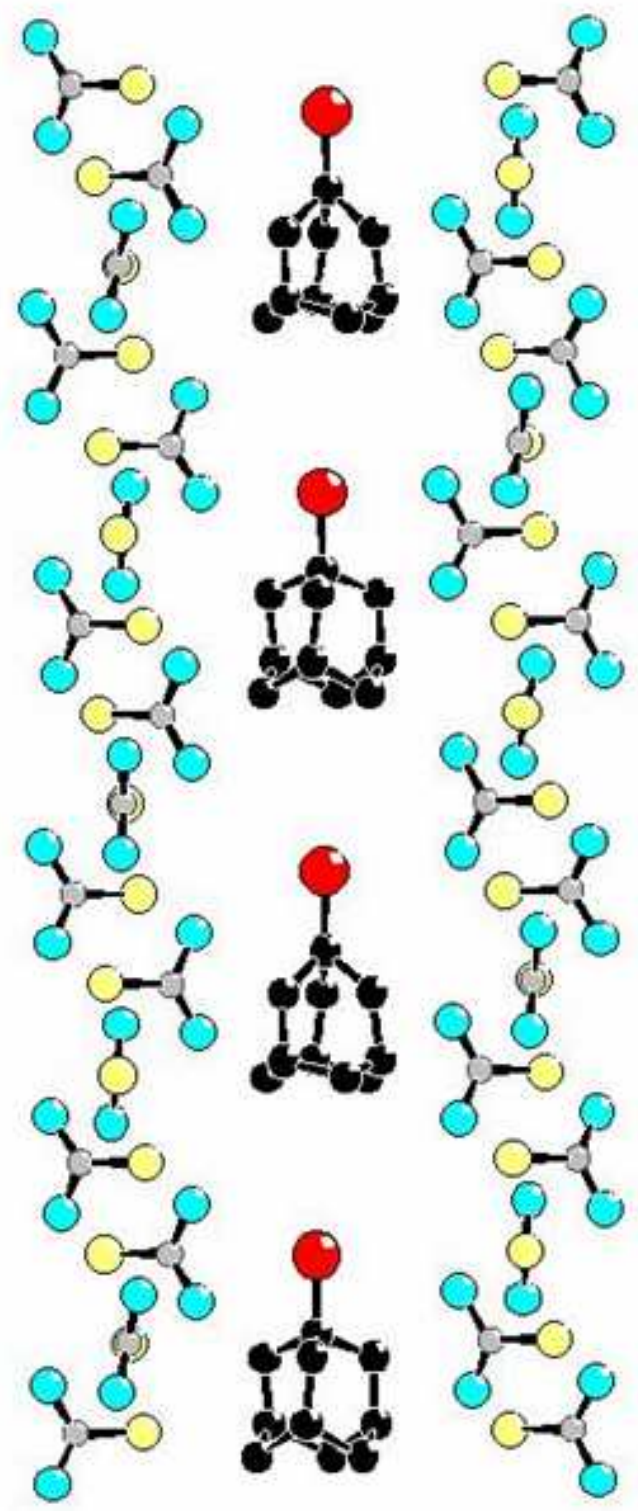

$50 \times 104 \mathrm{~mm}(150 \times 150 \mathrm{DPI})$ 


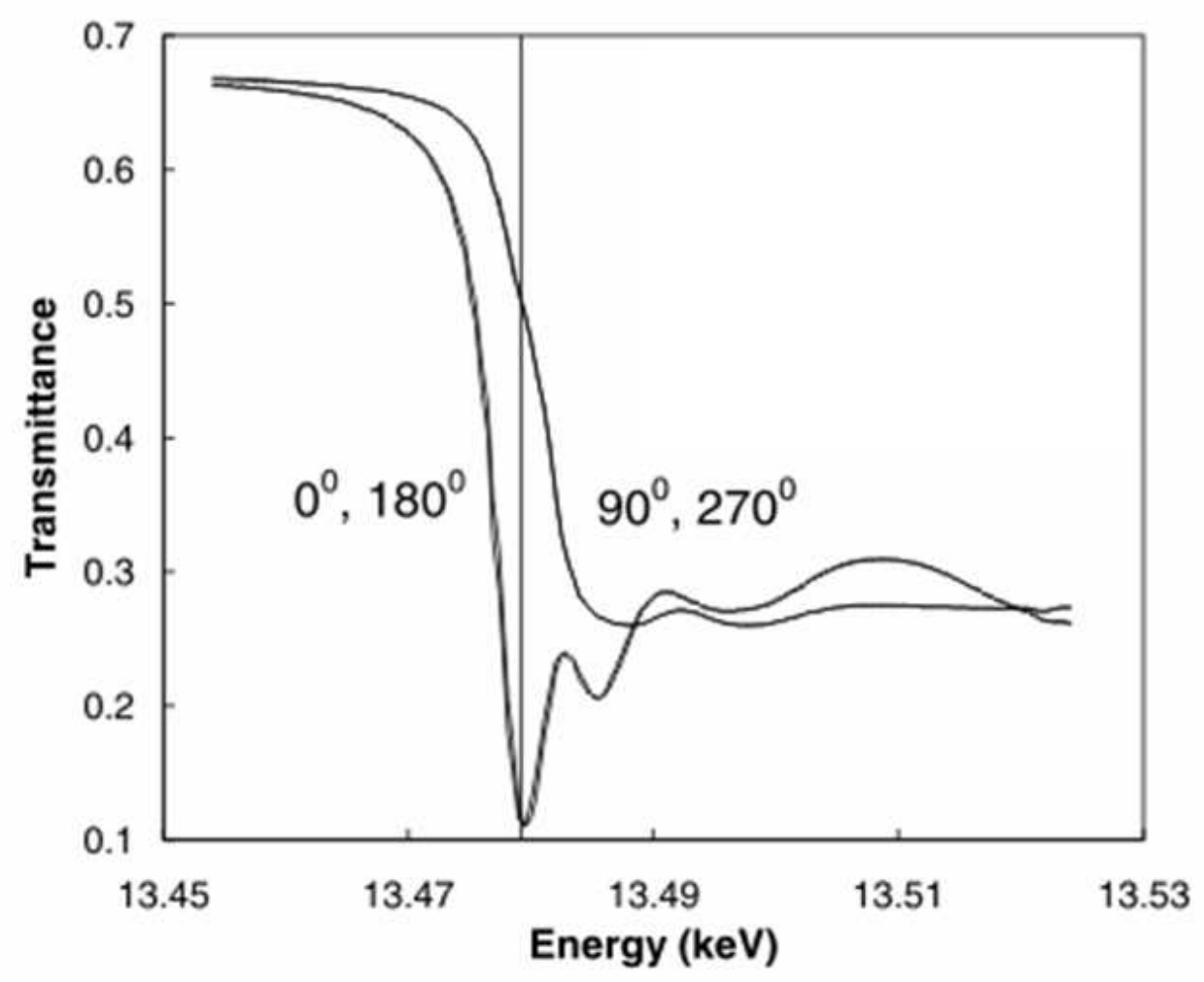

$111 \times 89 \mathrm{~mm}(150 \times 150 \mathrm{DPI})$ 
1

2

3

4

5

6

7

8

9

10

11

12

13

14

15

16

17

18

19

20

21

22

23

24

25

26

27

28

29

30

31

32

33

34

35

36

37

38

39

40

41

42

43

44

45

46

47

48

49

50

51

52

53

54

55

56

57

58

59

60

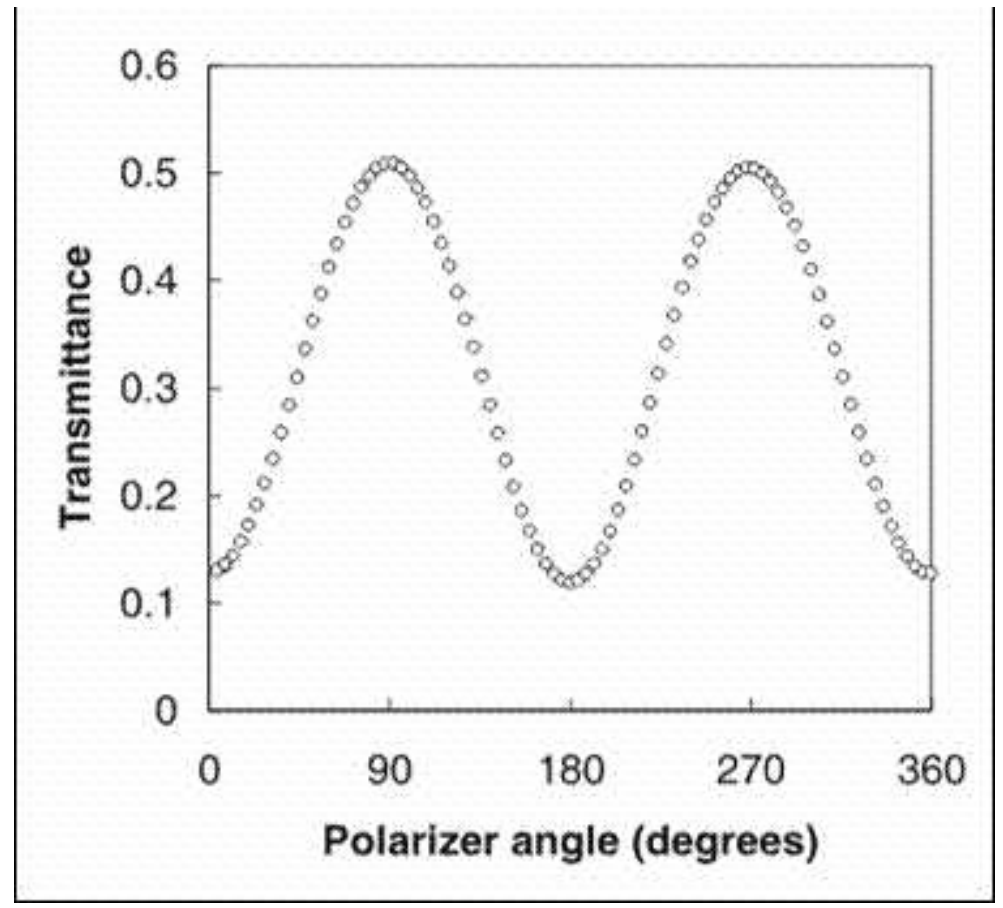

$99 \times 91 \mathrm{~mm}(96 \times 96 \mathrm{DPI})$ 